\title{
Nasal Carriage Rate, Antimicrobial Susceptibility Patterns and Associated Risk Factors of Methicillin- resistant Staphylococcus Aureus Among Prisoners in Dessie Town, Northeast Ethiopia
}

\section{Getinet Mamo}

Dessie Referral Hospital

\section{Getachew Ferede}

University of Gondar College of Medicine and Health Science

Teshome Belachew Eshete ( $\nabla$ tesh0926@gmail.com )

University of Gondar College of Medicine and Health Sciences https://orcid.org/0000-0003-4350-9803

\section{Research}

Keywords: MRSA, Antimicrobial Susceptibility, Nasal carriage, prison

Posted Date: September 2nd, 2020

DOI: https://doi.org/10.21203/rs.3.rs-66880/v1

License: (우 This work is licensed under a Creative Commons Attribution 4.0 International License. Read Full License 


\section{Abstract}

Background: The prevalence of Methicillin-resistant S. aureus (MRSA) within the general public has caused outbreaks in groups of people in close quarters such as military barracks, gyms, day care centres and correctional facilities. Correctional facilities are of particular importance for spreading MRSA, as inmates are often in close proximity and have limited access to hygienic products and clean clothing. In Ethiopia, there is a limited number of studies about methicillin-resistant $S$. aureus in the prison setting. Therefore, this study aimed to determine nasal carriages rate, antimicrobial susceptibility patterns, and associated risk factors of MRSA among prisoners in Dessie town, Northeast Ethiopia.

Methods: An institution-based cross-sectional study was conducted from January 1 to March 30/2020 on 329 prisoners. Nasal swabs were collected and processed on blood agar and Mannitol salt agar plates. The isolates were identified as $S$. aureus based on morphology, coagulase test, and mannitol salt agar fermentation. Disk diffusion antibiotic susceptibility tests were performed according to the guidelines of the Clinical and Laboratory Standards Institute. Methicillin-resistance by S. aureus was confirmed by using cefoxitin $(30 \mu \mathrm{g})$. Data entry and statistical analysis was done using SPSS Version 20.

Results: Out of the 329 prisoners, 115(35\%) carried S. aureus, of which 35/115 (30.4\%) were MRSA. Therefore, $10.64 \%$ of all prison inmates were identified as MRSA carriers. MRSA carriage rate was highest among the age groups of 24-34 and 35-45, which was 12 (34.3). Penicillin showed the highest rate of resistance among MRSA and MSSA isolates (100\%). The multi-drug resistance rate was very high (50.4\%). Among 115 S. aureus isolates, about 2(7.4\%) showed inducible Clindamycin resistance.

Conclusion: The high rate of nasal MRSA carriage among prisoners found in this study is alarming and highlights the need for adjusted infection control measures to prevent MRSA transmission within inmates or to the community during their discharge.

\section{Background}

Methicillin-resistant $S$. aureus (MRSA) is a Gram-positive bacterium that is genetically different from nonresistant strains. Resistance by S.aureus against methicillin has developed through horizontal gene transfer and natural selection (by mutation of a penicillin-binding protein) which is facilitated by the mecA gene, carried by Staphylococcal chromosome cassette (SCC) elements [1, 2]. Colonization with MRSA is linked with different recognized risk factors like; the history of hospitalization, admission to an intensive care unit, prolonged hospital stay, crowdedness, older age, invasive procedures, presence of wounds, and long-term antimicrobial therapy [3].

Despite the fact that MRSA is more common in both the healthcare institutions and the community, now a day, prison centers become a hot spot correctional institutions with the huge burden of MRSA colonization and infection. Less frequent discharge of prisoners may create greater opportunities for within-prison transmission of MRSA [4]. On the other hand, prisons have a fundamental role in transmission to the community by helping as important reservoirs for infections because, after release, most prisoners interact with the general population [5]. 
Data released by the U.S. Centers for Disease Control and Prevention (CDC) in 2019 showed that S. aureus infections are a major problem in the United States, with 119,000 infections and almost 20,000 deaths in 2017. In Louisiana, it is estimated that out of a 4,500,000 population, 1,500,000 are carriers of $S$. aureus and 45,000 are carriers of MRSA [6]. According to the report of the Pan-European Institute, MRSA was estimated to affect more than 150,000 patients annually in the European countries and is responsible for the expenditure of 380 million Euros in extra costs for healthcare systems [7]. Methicillin-resistant $S$. aureus is widely prevalent in most Asian countries too. For instance, multidrug-resistant MRSA are endemic in most hospitals, with an estimated prevalence from $28 \%$ in Hong Kong and Indonesia to greater than $70 \%$ in Korea [8].

In Africa, the burden of MRSA has remarkable variations across countries due to a lack of advanced laboratories, effective and systematic routine surveillance systems. Due to this, the prevalence of methicillin resistance in $S$. aureus isolates rises up to $82 \%$ (34). South Africa and central Africa are among regions where a high rate of MRSA prevalence reported with $24.4 \%$ and $40.4 \%$ respectively [9].

In Ethiopia, MRSA is spreading quickly both in hospital and community settings at a very alarming rate [10]. The study conducted in Jimma town, among prisoners, depicted that the overall prevalence of MRSA was $48 \%$ [11]. A study conducted among health workers of Dessie Referral Hospital showed the prevalence of MRSA was 12.7\% [12]. Another study conducted in Gondar town, among urban and rural elementary school children, showed that the prevalence of MRSA was 9.79\% [13]. The spread of MRSA in the health care system is a serious opportunity for transmission of MRSA outside the health care system. However, most of the studies related to MRSA colonization are limited to health care institutions. This is a relevant issue, since prison characteristics vary in different countries. Moreover, since those institutions harbor closed populations, it is important to know whether the prevalence of MRSA from those prisons similar to the prevalence in the hospitals or in the community. Therefore, this study will fill this gap and determine the prevalence of carriage, antimicrobial susceptibility patterns, and risk factors of MRSA among prisoners in Dessie town.

\section{Methods}

\section{Study Setting, Design, and Period}

An institution-based cross-sectional study was conducted in Dessie town prison center from January 1 to March 30/2020. Dessie town is located in Amhara Regional State, Northeast Ethiopia, $401 \mathrm{~km}$ far from Addis Ababa, and $471 \mathrm{~km}$ far from Bahir Dar. Based on the 2013 national census conducted by the Central Statistical Agency of Ethiopia (CSA) [14], Dessie town has a total population of 151,174, of whom 72,932 are men and 78,242 women [15]. During the study, about 1350 prisoners were jailed.

\section{Sample size and Sampling technique}


The sample size was determined by using a single population proportion formula by considering the prevalence of $48 \%$ [11], with a $95 \%$ confidence interval, a $5 \%$ margin of error, and with $10 \%$ no-response rate. A systematic random sampling technique was used to recruit 329 study percipients from the sampling frame formed by the registration number of prisoners. Prisoners with active upper respiratory tract infection, fever, recent nasal surgery, and use of nasal antimicrobial therapy were excluded.

\section{Data collection and laboratory methods}

\section{Data collection procedures}

A well-structured questionnaire was first prepared in English and then translated to Amharic language (local language). The second version of the questionnaire was retranslated into the original one by language experts to evaluate its consistency. The pre-tested final version of the questionnaire was used to collect data from study participants. Face- to -face interview technique was applied to collect data related to socio-demographic, clinical, and treatment-related variables as well as hygiene related factors.

\section{Sample collection and transportation}

Nasal swabs were collected by a trained laboratory technologist as per the collection and transportation procedures. A single sterile swab was used for each nostril. The sterile cotton swab was pre-moistened with sterile normal saline $(0.85 \% \mathrm{NaCl})$ before swabbing. The moistened cotton swab was introduced to the nostrils 2-3 cm and rotated 4-5 times both clockwise and anticlockwise before the withdrawal. The collected specimens were placed into Amies transport media and transported with an icebox to Amhara Public Health Institute, Dessie branch for further processing within 2 hours of collection $[16,17]$.

\section{Bacterial isolation and identification}

The nasal swab specimens were inoculated onto Sheep Blood Agar (Oxoid, LTD, UK) and Mannitol Salt Agar (Oxoid, England). All plates were incubated at $37^{\circ} \mathrm{C}$ for 24 hours under aerobic conditions. Preliminary identification of S.aureus was done based on the colony morphology on Mannitol and blood agar plates. Further confirmatory identification was done by Gram staining, catalase, mannitol fermentation, and coagulase tests following standard procedures [18].

\section{Antimicrobial susceptibility testing}

The antimicrobial susceptibility test of the isolates was determined according to the Kirby Bauer disc diffusion technique as illustrated by the Clinical Laboratory Standard Institute 2018 [19]. The bacterial suspension was made from pure colony of the test organism and matched with $0.5 \mathrm{McFarland}$ standards. Then, standardized culture suspension was swabbed with lawn technique onto the surface of Mueller- 
Hinton agar (MHA) plate. After 3 minutes, a set of 8 standard antimicrobial discs were then placed aseptically on the inoculated Mueller- Hinton agar plates and allowed to stand at room temperature for 15 minutes. All inoculated media were then incubated at $37^{\circ} \mathrm{C}$ aerobically. After $24 \mathrm{hrs}$ of incubation, the diameter of the zone of inhibition around each disk was measured with a ruler. Based on the zone of inhibition $(\mathrm{mm})$ of each antimicrobial disc, isolates were classified as resistant, intermediate, or susceptible following the standard interpretive chart of CLSI 2018 [19]. S. aureus ATCC 25923 (oxacillin-sensitive, and ATCC 33591 (oxacillin-resistant,) were used as controls in all experiments.

Methicillin-resistance by $S$. aureus was detected using surrogate antibiotics of $30 \mu \mathrm{g}$ cefoxitin discs by the Kirby-Bauer disk diffusion method. All the isolates were subjected to cefoxitin disc. Similarly, $0.5 \mathrm{McF}$. standard matched suspension of the isolates was prepared and inoculated on Mueller Hinton agar plate. Plates were incubated at $37^{\circ} \mathrm{C}$ for 24 hours and inhibition zone diameters $(\mathrm{mm})$ were interpreted based on CLSI 2018 guidelines [19]. Bacteria resisting for more than two drugs of the different classes were considered as "multi-drug resistant" [20]. An inhibition zone diameter of $\leq 21 \mathrm{~mm}$ was reported as methicillin-resistant and $\geq 22 \mathrm{~mm}$ was considered as methicillin-sensitive [19]. The following antimicrobial agents were tested; penicillin (10Units), erythromycin $(15 \mu \mathrm{g})$, clindamycin $(2 \mu \mathrm{g})$, trimethoprimsulphamethoxazole $(1.25 / 23.75 \mu \mathrm{g})$, tetracycline $(30 \mu \mathrm{g})$, ciprofloxacin $(5 \mu \mathrm{g})$, chloramphenicol $(30 \mu \mathrm{g})$, gentamicin $(10 \mu \mathrm{g})$, and cefoxitin $(30 \mu \mathrm{g})$.

\section{Data management and quality control}

\section{Data quality control}

The training was given for the data collector about data collection procedures and interviewing techniques for one day. The principal investigator, together with the supervisor, supervised the data collection. The pretest was carried out at the Kombolcha prison Centre.

\section{Laboratory quality control}

Pre-analytical, analytical, and post-analytical quality assurance was maintained [18].

\section{Data processing and analysis}

Initially, data were checked for completeness and coded. After coding, data were entered using EPI-info version 3.5.1 and exported to SPSS version 20 for further analysis. Frequency analysis was carried out to assess the prevalence of MRSA. Logistic regression analyses were done to determine factors associated with the carriage rate of MRSA. A variable with $p$-value $\leq 0.2$ in bivariate logistic regression was included in multivariate analysis. Crude and adjusted odds ratios were calculated to quantify the strength of association between MRSA carriage rate and risk factors. The $95 \%$ confidence interval was used and risk factors with $p$-value $<0.05$ in multivariate analyses were considered as statistically significant. 


\section{Ethical considerations}

Ethical clearance was obtained from the School of Biomedical and Laboratory Sciences, University of Gondar Ethical Review Committee. Ethical permission was obtained from Dessie prison centre office. Each study participant was informed about the purpose, methods of collection, anticipated benefit, and risk of study. Written informed consent was obtained from each study participant. Study subjects were identified using codes rather than using the actual name and unauthorized persons had no access to the collected data.

\section{Results}

\section{Socio demographic characteristics of study subjects}

Altogether, 329 prisoners were involved in this study. Of these, $288(87.5 \%)$ were males. The age of study participants was ranged from 17 to 77 years with a mean age of $35.27 \pm 10.72$ years. Among the study subjects, about 145/329 (44.1\%), completed primary school (Table 1).

Table 1

Socio-demographic characteristics of study participants among prisoners in Dessie, Northeast Ethiopia, 2020

\begin{tabular}{|c|c|c|c|}
\hline Variables & Category & Frequency (No) & Percentage (\%) \\
\hline \multirow[t]{2}{*}{ Gender } & Female & 41 & 12.5 \\
\hline & Male & 288 & 87.5 \\
\hline \multirow[t]{4}{*}{ Age } & $18-24$ & 67 & 20.4 \\
\hline & $25-34$ & 116 & 35.2 \\
\hline & $35-45$ & 90 & 27.4 \\
\hline & $>45$ & 56 & 17.0 \\
\hline \multirow[t]{5}{*}{ Level of education } & Unable to read and write & 52 & 15.8 \\
\hline & Informal education & 16 & 4.9 \\
\hline & Primary school & 145 & 44.1 \\
\hline & Secondary school & 82 & 24.9 \\
\hline & College/University & 52 & 15.8 \\
\hline
\end{tabular}

\section{Clinical and hygiene related characteristics of the study subjects}


Nearly half of the study subjects, $168 / 329$ (51.1\%), had a history of hospital/clinic visits. But, about $287 / 329$ (87.2\%) had no history of hospitalization, 324/329 (98.5\%) had no history of surgery, 308/329 $(93.8 \%)$ had no history of chronic disease, and about $268 / 329(81.5 \%)$ had no history of respiratory infections. Regarding the hygiene of the prisoners, about $48 / 329(14.6 \%)$ had a history of sharing beds with other prisoners and $83 / 329(25.2 \%)$ had a history of sharing personal items with others. The majority of study participants, 208/329 (63.2\%) clean their nostrils with other than handkerchiefs and Fingers, 314/329 (95.4\%) use soaps to wash their hands. About 182 (55.3\%) prisoners have been in a Crowded dorm (greater than 60/dorm) [Table 2]. 
Table 2

Clinical and hygiene related characteristics of study subjects among prisoners in Dessie, Northeast Ethiopia, 2020

\begin{tabular}{|c|c|c|c|}
\hline Variables & Category & $\begin{array}{l}\text { Frequency } \\
\text { (No) }\end{array}$ & $\begin{array}{l}\text { Percentage } \\
\text { (\%) }\end{array}$ \\
\hline \multirow[t]{2}{*}{ History of hospitals/clinics visit } & Yes & 168 & 51.1 \\
\hline & No & 161 & 48.9 \\
\hline \multirow[t]{2}{*}{ History of hospitalization } & Yes & 42 & 12.8 \\
\hline & No & 287 & 87.2 \\
\hline \multirow[t]{2}{*}{ History of surgery } & Yes & 5 & 1.5 \\
\hline & No & 324 & 98.5 \\
\hline \multirow[t]{2}{*}{ History of superficial skin lesion } & Yes & 59 & 17.9 \\
\hline & No & 270 & 82.1 \\
\hline \multirow[t]{2}{*}{ History of skin injury } & Yes & 24 & 7.3 \\
\hline & No & 305 & 92.7 \\
\hline \multirow[t]{2}{*}{ His. of contact with health workers } & Yes & 52 & 15.8 \\
\hline & No & 277 & 84.2 \\
\hline \multirow[t]{2}{*}{ History of treatment } & Yes & 127 & 38.6 \\
\hline & No & 202 & 61.4 \\
\hline \multirow[t]{2}{*}{ History of chronic disease } & Yes & 21 & 6.4 \\
\hline & No & 308 & 93.6 \\
\hline \multirow[t]{2}{*}{ History of respiratory infections } & Yes & 61 & 18.5 \\
\hline & No & 268 & 81.5 \\
\hline \multirow[t]{2}{*}{ Ambulatory history } & Yes & 2 & 0.6 \\
\hline & No & 327 & 99.4 \\
\hline \multirow{2}{*}{$\begin{array}{l}\text { Hospital admitted patients in prison } \\
\text { dormitory }\end{array}$} & Yes & 6 & 1.8 \\
\hline & No & 323 & 98.2 \\
\hline \multirow[t]{2}{*}{ Sharing of bed } & Yes & 48 & 14.6 \\
\hline & No & 281 & 85.4 \\
\hline \multirow[t]{2}{*}{ Sharing of personal items } & Yes & 83 & 25.2 \\
\hline & No & 246 & 74.8 \\
\hline Nostrils cleaning material & $\begin{array}{l}\text { handkerchiefs } \\
\text { Page } 8 / 22\end{array}$ & 64 & 19.5 \\
\hline
\end{tabular}




\begin{tabular}{|llll|}
\hline \multirow{2}{*}{ Washing hands } & Fingers & 57 & 17.3 \\
\cline { 2 - 4 } & other Means & 208 & 63.2 \\
\hline Shower frequency & Without Soap & 15 & 4.6 \\
\cline { 2 - 4 } & With Soap & 314 & 95.4 \\
\hline Number of prisoners/dorm & $\begin{array}{l}2 \text { to } 3 \text { times a } \\
\text { week }\end{array}$ & 192 & 58.4 \\
\cline { 2 - 4 } & One times a week & 137 & 41.6 \\
\hline Duration of stay in the prison & $21-40$ & 38 & 11.6 \\
\hline & $41-60$ & 109 & 33.1 \\
\hline & $>60$ & 182 & 55.3 \\
\hline & $>6$ month & 236 & 71.7 \\
\hline & $<6$ months & 93 & 28.3 \\
\hline
\end{tabular}

\section{Prevalence of $S$. aureus and MRSA}

Among a total of 329 prisoners, 115/329 (35\%) were colonized with S. aureus. Of 115 colonized individuals, $35 / 115$ (30.4\%) were colonized with MRSA. Therefore, $35 / 32910.64 \%$ of all prison inmates were identified as MRSA carriers. About 44/116 (39.9\%) of $S$. aureus was isolated from the age group of 25-34 years whereas about $12 / 35$ (34.3\%) of MRSA was isolated from the age group of 25-34 and 35-45 years. Nasal carriage of $S$. aureus among female and male prisoners were $16(13.9 \%)$ and $99(86.1 \%)$, respectively. The isolation rate of MRSA was higher among males 29 (82.9\%) as compared to that of females $6(17.1 \%)$ [Table 3]. 
Table 3

Prevalence of S. aureus and MRSA among prisoners in Dessie, Northeast, Ethiopia, 2020.

\begin{tabular}{|c|c|c|c|c|c|}
\hline \multirow[t]{3}{*}{ Variables } & \multirow[t]{3}{*}{ Categories } & \multirow{3}{*}{$\begin{array}{l}\text { S. aureus } \\
\text { Positive }(n=115) \mathrm{N} \\
(\%)\end{array}$} & \multicolumn{3}{|c|}{ MRSA } \\
\hline & & & Negative & \multirow{2}{*}{$\begin{array}{l}\text { Positive }(\mathrm{n}=35) \\
\mathrm{N}(\%)\end{array}$} & \multirow{2}{*}{$\begin{array}{l}\text { Negative }(n=80) \\
N(\%)\end{array}$} \\
\hline & & & (1) & & \\
\hline \multirow{3}{*}{ Gender } & & & & & \\
\hline & Female & 16(13.9) & $25(11.7 \%)$ & $6(17.1)$ & 10 (12.5) \\
\hline & Male & $99(86.1)$ & $189(88.3 \%)$ & $29(82.9)$ & 70 (87.5) \\
\hline \multirow[t]{4}{*}{ Age } & $18-24$ & 19 (16.5) & $48(22.4 \%)$ & $3(8.6)$ & $16(20)$ \\
\hline & $25-34$ & $44(38.3)$ & 72 (33.4\%) & 12 (34.3) & $32(40)$ \\
\hline & $35-45$ & $31(30)$ & 59 (27.6\%) & $12(34.3)$ & $19(23.8)$ \\
\hline & $>45$ & 21(18.3) & 35 (16.4\%) & $8(22.9)$ & 13 (16.3) \\
\hline
\end{tabular}

\section{Factors associated with MRSA colonization}

After bivariate logistic regression analysis, age $(P=0.096)$, history of visiting hospitals/clinics $(P=0.006)$, history of superficial skin lesion $(P=0.000)$, history of treatment $(P=0.001)$, sharing of bed $(P=0.067)$, sharing of personal items $(P=0.000)$, types of nostrils cleaning materials $(P=0.0136)$, shower frequency $(P=0.004)$, number of prisoners per dorm $(P=0.115)$, body mass index $(P=0.068)$ were included in a multivariate logistic regression analysis.

Of the clinical risk factors, having a history of superficial skin lesion [AOR: 8,152 (95\% Cl: 2.303, 28.861); $P=0.001$ ] and history of treatment [AOR: 8.008 (95\% Cl 2.021, 31.724); $P=0.003$ ] were positively associated with MRSA nasal colonization. Regarding hygiene, the sharing of personal items [AOR: $4.016(95 \% \mathrm{Cl} 1.330$, 12.122); $P=0.014$ ] was positively associated with MRSA nasal colonization. However, cleaning nostrils other than handkerchiefs and fingers [AOR: $0.174(95 \% \mathrm{Cl} 0.042,0.721) ; P=0.016]$ was negativity associated with nasal carriage of MRSA. The association between shower frequency, number of prisoners per dorm and MRSA was not significant [Table 4]. 
Table 4

Bivariate and Multivariate analysis of MRSA among prisoners in Dessie, Northeast, Ethiopia, 2020

\begin{tabular}{|c|c|c|c|c|c|c|c|}
\hline \multirow[t]{2}{*}{ Characteristics } & \multirow[t]{2}{*}{ Categories } & \multicolumn{2}{|c|}{ MRSA } & \multirow{2}{*}{)$^{\mathrm{COR}(95 \% \mathrm{Cl}}$} & \multirow{2}{*}{$\begin{array}{l}P \\
\text { value }\end{array}$} & \multirow{2}{*}{$\begin{array}{l}\text { AOR [95\% } \\
\text { C.I.] }\end{array}$} & \multirow{2}{*}{$\begin{array}{l}P \\
\text { value }\end{array}$} \\
\hline & & $\begin{array}{l}\text { Yes } \\
(n=35) \\
N(\%)\end{array}$ & $\begin{array}{l}\text { No } \\
(n=80) \\
N(\%)\end{array}$ & & & & \\
\hline \multirow[t]{2}{*}{ Sex } & Female & $6(38)$ & $\begin{array}{l}10 \\
(63)\end{array}$ & 1 (ref.) & & & \\
\hline & Male & $29(29)$ & $\begin{array}{l}70 \\
(71)\end{array}$ & $\begin{array}{l}0.69(0.230- \\
2.076)\end{array}$ & 0.712 & & \\
\hline \multirow[t]{4}{*}{ Age } & $18-24$ & $3(16)$ & $\begin{array}{l}16 \\
(84)\end{array}$ & 1 (ref.) & & 1 (ref.) & \\
\hline & $25-34$ & $12(27)$ & $\begin{array}{l}32 \\
(73)\end{array}$ & $\begin{array}{l}2.0(0.493- \\
8.113)\end{array}$ & 0.332 & $\begin{array}{l}1.157 \\
(0.155- \\
8.650)\end{array}$ & 0.887 \\
\hline & $35-45$ & $12(39)$ & $\begin{array}{l}19 \\
(61)\end{array}$ & $\begin{array}{l}3.368 \\
(0.807- \\
14.066)\end{array}$ & 0.096 & $\begin{array}{l}2.790 \\
(0.353- \\
22.075)\end{array}$ & 0.331 \\
\hline & $>45$ & $8(38)$ & $\begin{array}{l}13 \\
(62)\end{array}$ & $\begin{array}{l}3.282 \\
(0.721- \\
14.937)\end{array}$ & 0.124 & $\begin{array}{l}0.912 \\
(0.095- \\
8.776)\end{array}$ & 0.936 \\
\hline \multirow[t]{2}{*}{$\begin{array}{l}\text { History of } \\
\text { visiting } \\
\text { hospitals }\end{array}$} & Yes & $9(39)$ & $14(61)$ & $\begin{array}{l}4.9(1.577- \\
15.243)\end{array}$ & 0.006 & $\begin{array}{l}0.728 \\
(0.122- \\
4.362)\end{array}$ & 0.729 \\
\hline & No & $26(28)$ & $\begin{array}{l}66 \\
(72)\end{array}$ & 1(Ref.) & & & \\
\hline \multirow[t]{2}{*}{$\begin{array}{l}\text { History of } \\
\text { hospitalization }\end{array}$} & Yes & $9(39)$ & $14(61)$ & $\begin{array}{l}1.632 \\
(0.630- \\
4.230)\end{array}$ & 0.314 & & \\
\hline & No & $26(28)$ & $\begin{array}{l}66 \\
(72)\end{array}$ & 1(Ref.) & & & \\
\hline \multirow[t]{2}{*}{$\begin{array}{l}\text { History of } \\
\text { surgery }\end{array}$} & Yes & $1(25)$ & $3(75)$ & $\begin{array}{l}0.755 \\
(0.076- \\
7.521)\end{array}$ & 0.811 & & \\
\hline & No & $34(31)$ & $77(67)$ & 1(Ref.) & & & \\
\hline \multirow[t]{2}{*}{$\begin{array}{l}\text { History of skin } \\
\text { lesion }\end{array}$} & Yes & $21(57)$ & $\begin{array}{l}16 \\
(43)\end{array}$ & $\begin{array}{l}6.0(2.513- \\
14.326)\end{array}$ & 0.000 & $\begin{array}{l}8.152 \\
(2.303- \\
28.861)\end{array}$ & $0.001^{*}$ \\
\hline & No & $14(18)$ & $\begin{array}{l}64 \\
(82)\end{array}$ & 1(Ref.) & & 1(Ref.) & \\
\hline $\begin{array}{l}\text { History of skin } \\
\text { injury }\end{array}$ & Yes & $2(18)$ & $9(82)$ & $\begin{array}{l}0.478 \\
(0.098- \\
2.337)\end{array}$ & 0.362 & & \\
\hline
\end{tabular}




\begin{tabular}{|c|c|c|c|c|c|c|c|}
\hline & No & $33(32)$ & $\begin{array}{l}71 \\
(68)\end{array}$ & 1(Ref.) & & & \\
\hline \multirow[t]{2}{*}{$\begin{array}{l}\text { contact with } \\
\text { health workers }\end{array}$} & Yes & 7 (32) & $\begin{array}{l}15 \\
(68)\end{array}$ & $\begin{array}{l}1.083 \\
(0.398- \\
2.947)\end{array}$ & \multicolumn{3}{|l|}{0.875} \\
\hline & No & $28(30)$ & $\begin{array}{l}65 \\
(70)\end{array}$ & 1(Ref.) & & & \\
\hline \multirow[t]{2}{*}{$\begin{array}{l}\text { History of } \\
\text { antibiotics }\end{array}$} & Yes & $29(43)$ & $\begin{array}{l}38 \\
\text { (57) }\end{array}$ & $\begin{array}{l}5.342 \\
(2.000- \\
14.269)\end{array}$ & 0.001 & $\begin{array}{l}8.008 \\
(2.021- \\
31.724)\end{array}$ & $0.003^{*}$ \\
\hline & No & $6(13)$ & $\begin{array}{l}42 \\
(86)\end{array}$ & 1(Ref.) & & & \\
\hline \multirow[t]{2}{*}{$\begin{array}{l}\text { History of } \\
\text { chronic } \\
\text { disease }\end{array}$} & Yes & $1(14)$ & $6(86)$ & $\begin{array}{l}0.363 \\
(0.042- \\
3.1320\end{array}$ & 0.357 & & \\
\hline & No & $34(32)$ & 74(69) & 1(Ref.) & & & \\
\hline \multirow[t]{2}{*}{$\begin{array}{l}\text { His. of respir. } \\
\text { Infect. }\end{array}$} & Yes & 10(36) & 18(64) & $\begin{array}{l}1.378 \\
(0.559- \\
3.395)\end{array}$ & 0.486 & & \\
\hline & No & $25(29)$ & $62(71)$ & 1(Ref.) & & & \\
\hline \multirow{2}{*}{$\begin{array}{l}\text { Ambulatory } \\
\text { history }\end{array}$} & Yes & $0(0)$ & $1(100)$ & 0.000 & 1.000 & & \\
\hline & No & $35(31)$ & 79(69) & 1(Ref.) & & & \\
\hline \multirow[t]{2}{*}{$\begin{array}{l}\text { Sharing of bed } \\
\text { with other } \\
\text { people }\end{array}$} & Yes & $4(18)$ & 18(82) & $\begin{array}{l}0.444 \\
(0.138- \\
1.426)\end{array}$ & 0.173 & $\begin{array}{l}0.389 \\
(0.084- \\
1.799)\end{array}$ & 0.227 \\
\hline & No & $31(33)$ & $62(67)$ & 1(Ref.) & & (Ref.) & \\
\hline \multirow[t]{2}{*}{$\begin{array}{l}\text { Sharing of } \\
\text { items }\end{array}$} & Yes & $25(52)$ & $23(48)$ & $\begin{array}{l}6.196 \\
(2.573- \\
14.919)\end{array}$ & 0.000 & $\begin{array}{l}4.016 \\
(1.330- \\
12.122)\end{array}$ & $0.014^{*}$ \\
\hline & No & $10(15)$ & $57(85)$ & 1(Ref.) & & 1(Ref.) & \\
\hline \multirow{3}{*}{$\begin{array}{l}\text { Nosrils } \\
\text { Cleaning } \\
\text { materials }\end{array}$} & Handkerchif & $11(46)$ & $13(54)$ & 1(Ref.) & & 1(Ref.) & \\
\hline & Fingers & $6(43)$ & $8(57)$ & $\begin{array}{l}0.886 \\
(0.235- \\
3.347)\end{array}$ & 0.136 & $\begin{array}{l}0.531 \\
(0.087- \\
3.222)\end{array}$ & 0.491 \\
\hline & other & $18(23)$ & $59(77)$ & $\begin{array}{l}0.361(0.138- \\
0.942)\end{array}$ & 0.037 & $\begin{array}{l}0.174 \\
(0.042- \\
0.721)\end{array}$ & $0.016^{*}$ \\
\hline \multirow{2}{*}{$\begin{array}{l}\text { Washing } \\
\text { hands }\end{array}$} & W/out Soap & $0(0)$ & $6(100)$ & 0.00 & 0.999 & & \\
\hline & With Soap & $35(32)$ & $74(68)$ & 1(Ref.) & & & \\
\hline Shower & 2-3/ week & $8(16)$ & $42(84)$ & 1(Ref.) & & & \\
\hline
\end{tabular}




\begin{tabular}{|c|c|c|c|c|c|c|c|}
\hline trequency & 1/ week & $27(42)$ & $38(59)$ & $\begin{array}{l}3.73(1.512- \\
9.201)\end{array}$ & 0.004 & $\begin{array}{l}3.423 \\
(0.977- \\
11.995)\end{array}$ & 0.054 \\
\hline \multirow{3}{*}{$\begin{array}{l}\text { Number of } \\
\text { prisoners per } \\
\text { dorm }\end{array}$} & $21-40$ & $1(10)$ & $9(90)$ & & & 1(Ref.) & \\
\hline & $41-60$ & $3(13)$ & $20(87)$ & $\begin{array}{l}1.350 \\
(0.123- \\
14.822)\end{array}$ & 0.806 & $\begin{array}{l}0.734 \\
(0.041- \\
13.253)\end{array}$ & 0.834 \\
\hline & $>60$ & $31(38)$ & $51(62)$ & $\begin{array}{l}5.471 \\
(0.661- \\
45.288)\end{array}$ & 0.115 & $\begin{array}{l}4.176 \\
(0.411- \\
42.413)\end{array}$ & 0.227 \\
\hline \multirow{2}{*}{$\begin{array}{l}\text { Duration of } \\
\text { stay in this } \\
\text { prison }\end{array}$} & $>6$ months & $29(33)$ & $59(67)$ & $\begin{array}{l}1.72(0.626- \\
4.725)\end{array}$ & 0.293 & & \\
\hline & $<6$ months & $6(22)$ & 21(78) & 1(Ref.) & & & \\
\hline \multirow[t]{3}{*}{$\begin{array}{l}\text { Body mass } \\
\text { index }\end{array}$} & $<18.5$ & $1(13)$ & $7(88)$ & $\begin{array}{l}0.329 \\
(0.041- \\
2.929)\end{array}$ & 0.329 & $\begin{array}{l}3.901(0.141- \\
107.57)\end{array}$ & 0.421 \\
\hline & $18.5-24.9$ & $29(29 \%)$ & $\begin{array}{l}70 \\
(71 \%)\end{array}$ & 1(Ref.) & & 1 (ref.) & \\
\hline & $25-29.9+-$ & $5(63 \%)$ & $\begin{array}{l}3 \\
(38 \%)\end{array}$ & $\begin{array}{l}3.966 \\
(0.889- \\
17.695)\end{array}$ & 0.068 & $\begin{array}{l}0.719 \\
(0.051- \\
10.041)\end{array}$ & 0.806 \\
\hline
\end{tabular}

\section{Antimicrobial susceptibility patterns of $S$. aureus}

All 115 S. aureus isolates were tested against 9 commonly used antimicrobials. The highest sensitivity was observed for clindamycin $(90.4 \%, 104)$ followed by chloramphenicol $(84.3 \%, 97)$ ciprofloxacillin $(83.5 \%, 96)$, tetracycline $(77.4 \%, 89)$, erythromycin $(82.6 \%, 95)$, and gentamicin $(76.5 \%, 88)$. However, all S.aureus isolates were found to be resistant to Penicillin $(100 \%, 115)$. Based on Cefoxitin susceptibility test result, 115 S. aureus isolates were grouped in to MRSA $(30.4 \%, 35)$ and methicillin sensitive $S$. aureus (MSSA) $(69.6 \%, 80)$. Of 35 MRSA isolates, about $29(82.9 \%)$ were sensitive for clindamycin, followed by $26(74.3 \%)$, gentamicin, 25(71.3\%), and tetracycline, 24(68.4\%) [Table 5]. 
Table 5

Antimicrobial susceptibility of S. aureus isolates among prisoners in Dessie, Northeast, Ethiopia, 2020

\begin{tabular}{|c|c|c|c|c|}
\hline \multicolumn{2}{|l|}{ Antimicrobials } & \multirow{2}{*}{$\begin{array}{l}\text { S.aureus isolates } \\
(n=115) N(\%)\end{array}$} & \multirow{2}{*}{$\begin{array}{l}\text { MRSA (n=35) } \\
N(\%)\end{array}$} & \multirow{2}{*}{$\begin{array}{l}\text { MSSA }(n=80) \\
N(\%)\end{array}$} \\
\hline & & & & \\
\hline \multirow[t]{2}{*}{ Penicillin } & Susceptible & $0(0.0)$ & $0(0.0)$ & $0(0.0)$ \\
\hline & Resistant & $115(100)$ & $35(100)$ & $80(100)$ \\
\hline \multirow[t]{2}{*}{ Cefoxitin } & Susceptible & $80(69.6)$ & $0(0.0)$ & $80(100)$ \\
\hline & Resistant & $35(30.4)$ & $35(100)$ & $0(0.0)$ \\
\hline \multirow[t]{2}{*}{ trimethoprim-sulfamethoxazole } & Susceptible & $84(73.0)$ & $21(60.0)$ & $63(78.8)$ \\
\hline & Resistant & $31(27.0)$ & $14(40.0)$ & $17(21.2)$ \\
\hline \multirow[t]{2}{*}{ Ciprofloxacillin } & Susceptible & $96(83.5)$ & $23(65.7)$ & 73(91.2) \\
\hline & Resistant & $19(16.5)$ & $12(34.3)$ & $7(8.8)$ \\
\hline \multirow[t]{2}{*}{ Tetracycline } & Susceptible & $89(77.4)$ & $25(71.4)$ & $64(80.0)$ \\
\hline & Resistant & $26(22.6)$ & 10(28.6) & $16(20.0)$ \\
\hline \multirow[t]{2}{*}{ Chloramphenicol } & Susceptible & $97(84.3)$ & $24(68.6)$ & $73(91.2)$ \\
\hline & Resistant & $18(15.724)$ & 11(31.4) & $7(8.8)$ \\
\hline \multirow[t]{2}{*}{ Gentamycin } & Susceptible & $88(76.5)$ & $26(74.3)$ & $62(77.5)$ \\
\hline & Resistant & $27(23.5)$ & $9(25.7)$ & $18(22.5)$ \\
\hline \multirow[t]{2}{*}{ Erythromycin } & Susceptible & $88(76.5)$ & $23(65.7)$ & $65(81.2)$ \\
\hline & Resistant & $27(23.5)$ & $12(34.3)$ & $15(18.8)$ \\
\hline \multirow[t]{2}{*}{ Clindamycin } & Susceptible & 104(90.4) & $29(82.9)$ & 75(93.8) \\
\hline & Resistant & $11(9.6)$ & $6(17.1)$ & $5(6.2)$ \\
\hline
\end{tabular}

\section{Multidrug resistance pattern of S.aureus}

The overall multidrug resistance (MDR) rate of bacterial isolates in this study was $50.4 \%$. About $16.5 \%$ of the bacterial isolates showed MDR to four antimicrobials followed by $7.8 \%$ to five antimicrobials [Table 6 ]. 
Table 6

Multidrug resistance pattern of $S$. aureus isolates among prisoners in Dessie, Northeast, Ethiopia, 2020

\begin{tabular}{|c|c|c|}
\hline Panel of antimicrobials & MDR pattern & Resistance strains No. (\%) \\
\hline \multirow[t]{8}{*}{ Three antimicrobials } & PEN/SXT/CIP & $3(2.6 \%)$ \\
\hline & PEN/SXT/ER & $5(4.3 \%)$ \\
\hline & PEN/SXT/GM & $3(2.6 \%)$ \\
\hline & PEN/SXT/TE & $4(3.5 \%)$ \\
\hline & $\mathrm{PEN} / \mathrm{ER} / \mathrm{CD}$ & $6(5.2 \%)$ \\
\hline & PEN/TE/GM & $5(4.3 \%)$ \\
\hline & CXT/PEN/CIP/C & $1(0.9 \%)$ \\
\hline & CXT/PEN/CIP/GM & $1(0.9 \%)$ \\
\hline \multirow[t]{11}{*}{ Four antimicrobials } & CXT/PEN/SXT/C & $2(1.7 \%)$ \\
\hline & CXT/PEN/SXT/CIP & $2(1.7 \%)$ \\
\hline & CXT/PEN/TE/ER & $3(2.6 \%)$ \\
\hline & CXT/PEN/TE/GM & $2(1.7 \%)$ \\
\hline & $\mathrm{PEN} / \mathrm{C} / \mathrm{ER} / \mathrm{CD}$ & $1(0.9 \%)$ \\
\hline & PEN/SXT/CIP/ER & $1(0.9 \%)$ \\
\hline & PEN/SXT/TE/C & $1(0.9 \%)$ \\
\hline & PEN/SXT/TE/ER & $1(0.9 \%)$ \\
\hline & $\mathrm{PEN} / \mathrm{GM} / \mathrm{ER} / \mathrm{CD}$ & $1(0.9 \%)$ \\
\hline & $\mathrm{PEN} / \mathrm{TE} / \mathrm{C} / \mathrm{GM}$ & $1(0.9 \%)$ \\
\hline & PEN/TE/ER/CD & $1(0.9 \%)$ \\
\hline \multirow[t]{6}{*}{ Five antimicrobials } & CXT/PEN/SXT/C/ER & $1(0.9 \%)$ \\
\hline & CXT/PEN/SXT/CIP/GM & $1(0.9 \%)$ \\
\hline & CXT/PEN/SXT/ER/CD & $1(0.9 \%)$ \\
\hline & CXT/PEN/SXT/TE/C & $3(2.6 \%)$ \\
\hline & CXT/PEN/SXT/TE/ER & $1(0.9 \%)$ \\
\hline & PEN/COT/CIP/ER/CD & $1(0.9 \%)$ \\
\hline \multirow[t]{2}{*}{ Six antimicrobials } & CXT/PEN/C/ER/GM/CD & $1(0.9 \%)$ \\
\hline & CXT/PEN/CIP/C/ER/GM & $1(0.9 \%)$ \\
\hline
\end{tabular}




\begin{tabular}{|lll|} 
& CXT/PEN/CIP/C/GM/CD & $1(0.9 \%)$ \\
\cline { 2 - 3 } & CXT/PEN/SXT/CIP/ER/CD & $2(1.7 \%)$ \\
\hline Seven antimicrobials & CXT/PEN/CIP/C/GM/ER/CD & $1(0.9 \%)$ \\
\hline
\end{tabular}

Note: $C X T=$ Cefoxitin, Pen=Penicillin, $S X T=$ trimethoprim-sulfamethoxazole, CIP=Ciprofloxacin, $C=$ Chloramphenicol, $T e=$ Tetracycline, $G M=$ Gentamycin, Ery= Erythromycin, $C D=$ Clindamycin

\section{Prevalence of Inducible Clindamycin Resistance}

Among 115 S. aureus isolates, about 2(7.4\%) showed inducible Clindamycin resistance [Table 7].

Table 7

Prevalence of Inducible Clindamycin Resistance of $S$. aureus among prisoner in Dessie, Northeast, Ethiopia, 2020

\begin{tabular}{|lll|}
\hline \multirow{2}{*}{ Erythromycin resistance $(\mathrm{n}=27)$} & \multicolumn{2}{l|}{ Clindamycin } \\
\cline { 2 - 3 } & D-test positive & D-test negative \\
\cline { 2 - 3 } & $2(7.4 \%)$ & $12(44.4 \%)$ \\
\hline
\end{tabular}

\section{Discussion}

S. aureus is a common bacterium that can colonize many parts of the body, mostly the nasal cavity. Approximately $20-30 \%$ of the world's human population is persistently colonized by $S$. aureus. Colonization rates of $S$. aureus are different in different risk groups. However, the highest frequency of carriage is observed among high-risk groups, like children and inmates in correctional institutions [21, 22]. Studies have indicated that nasal colonization of $S$. aureus has a key role in developing subsequent infections [23].

In the present study, the overall prevalence of $S$. aureus colonization among prisoners was $35 \%$. The nasal carriage rate of $S$.aureus observed in this study was smaller than the findings of the study conducted in USA maximum-security prisons (54.4\%) and Jimma prison center, Ethiopia (50\%) [24, 11]. However, the carriage rate of $S$. aureus in the present study was higher than studies done in Brazil (16.5\%) (30). The differences might reflect the variations in isolation techniques, the sample size, time of study period, population characteristics, geographical distribution, prevention and control policies, and duration of stay in correctional facilities. This study is in line with a study conducted in Texas county jail inmates (28.5\%) [11].

The overall nasal carriage rate of MRSA among prisoners was $10.64 \%$. Of the total 115 S. aureus isolates, the prevalence of MRSA was $30.4 \%$ which was similar to the result of a study conducted in South Africa (30.9\%) [25] and Saud Arabia (32\%) [26]. However, this finding was lower than the reports of other studies, such as MRSA carriage rate of $41 \%$ in the manila city jail, Philippine[11], $54 \%$ in the USA[27], and $48 \%$ in Jimma town correctional facility, Ethiopia [28]. The smaller carriage rate of MRSA in the present study might be due to the variation in isolating techniques, study subjects, population characteristics, and length of study time. 
In this study, the high nasal colonization of MRSA among prisoners was attributed to the contribution of different risk factors. The risk factors mainly identified from this study after doing multivariate logistic regression analysis were; having a history of superficial skin lesions, a history of antibiotic use, sharing of personal items, and ways of cleaning nostrils. According to the results of the present study, the nasal colonization of MRSA was predominant in participants with a history of superficial skin lesion in the previous one year (56.8\%) than prisoners without a history of superficial skin lesions (17.9\%). The data showed that it was 6 times more likely for prisoners with a history of superficial skin lesions to have carriage of MRSA than prisoners without it, $(p<0.001)$. The findings of this study were comparable with the report of the study conducted in the Los Angeles County Jail $(p=0.001)$ [29]. The possible reason might be due to the fact that prisoners with superficial skin lesion may receive empirical therapy or indiscriminate use of antibiotics to treat the infection which has a key role for the development of resistant strains like MRSA.

MRSA nasal carriage rate was the highest among prisoners with a history of treatment compared to prisoners without a history of treatment [29 $(43.3 \%)$ VS $6(12.5 \%) ; p=0.003]$. It is consistent with the reports of the study conducted in some community-based studies on healthy children living in Kashan/Iran, Netherlands, and Jimma, Ethiopia [11, 30,31]. This can be explained by the fact that the prior and continuous use of antibiotics can create good competitive advantage for the development of resistant bacteria and increment of carriage of persistent HA-MRSA.

The association between MRSA colonization and sharing personal items with other inmates in this study was statistically significant [AOR: 6.196 (95\% Cl 2.573-14.919); $p=0.014$ ]. It was inconsistent with the study conducted in the Los Angeles county jail with the results of an insignificant association [29]. This finding showed the possible advantages of sharing personal items such as towels, shoes, soaps, washcloths, and house shoes in prison inmates for the continuous transmission of MRSA from the carrier inmate to the healthy one. Additionally MRSA nasal colonization was negatively associated with the ways of cleaning nostrils. However, there was no study among prisoners which showed the nostril hygiene practices of inmates.

Antimicrobial resistance has been recognized as an emerging worldwide problem both in developed and developing countries. In the current study, all S. aureus isolates were $100 \%$ resistant to penicillin antibiotic which was consistent with the study conducted among children in Nigeria (100\%), in Bahir Dar town (100\%), and Gondar(100\%), Ethiopia [11, 13,32]. However, higher Sensitivity was observed to Clindamycin, Chloramphenicol, and Ciprofloxacillin with the sensitivity rate of $90.4 \%, 84.3 \%$, and $83.5 \%$ respectively. This Result was comparable with findings reported in Bahir Dar [32] and Pakistan [33].

In the present study, the frequency of inducible clindamycin resistance was $2 / 115$ (1.74\%). This finding was inconsistent with findings reported in Brunei Darussalam (15.7\%) [34]. This difference may be resulted in variation of geographical area.

\section{Conclusion}


Nasal colonization with MRSA is the most common source for the occurrence of subsequent infection of the host. The findings of this study demonstrated that the carriage rate of MRSA was high. Having a history of superficial skin lesions, history of treatment, history of sharing personal items and types of nostrils cleaning materials were significantly associated with MRSA colonization. The rise of the prevalence of nasal colonization may increase the likelihood of MRSA associated infections and outbreaks. Effective control measures are required for preventing possible cross-colonization and infection.

According to the antimicrobial susceptibility test results of the current study, all $S$. aureus isolates were resistant to Penicillin. But, greater sensitivity to Clindamycin was observed and it could be the major candidate drug for the treatment of MRSA associated infection in a correctional facility. However, antimicrobial susceptibility test prior to empirical prescription of drugs is mandatory due to the rise of the current trends in drug resistance. A significant rate of MDR was shown by the isolates so that infection control and prevention policies which are important for monitoring the effectiveness of antimicrobial stewardship programs to reduce the burden of drug resistance is essential. Moreover, since those institutions harbor closed populations, it is important to know whether MRSA from those prisons have molecular features similar to those of strains originating in the hospital or in the community.

\section{Abbreviations}

CLSI: Clinical and Laboratory Standard Institute, HA-MRSA: Hospital Acquired Methicillin Resistant $S$. aureus. MHA: Mueller Hinton Agar, MRSA: Methicillin Resistance S. aureus, MSA: Mannitol Salt Agar, SBA: Sheep Blood Agar

\section{Declarations}

\section{Ethics approval and consent to participate}

An ethical clearance letter was obtained from the Departmental Research and Ethics Review Committee of school of biomedical laboratory science. The reference number of the ethical letter was "Ref noSBMLS/2445/2020". This ethical letter was obtained from Mr. Mekonnen Girma (mekonnen2302@cmail.com), Markos Negash (markosnegash@yahoo.com) and Bamilaku Enawgaw (bamlak21@gmail.com).Written consent was obtained from participants and informed as their participation was voluntary. Study participants were also informed about the purpose of the study. Confidentiality was maintained at all levels of the study. In addition, study participants involvement was based on a voluntary basis and participants who were unwilling to take part in the study and those who need to quit their participation at any stage were informed to do so without any restriction. Consent to participate was obtained from the study participants.

\section{Consent for publication}

All authors read the manuscript and have provided their consent to publish. 


\section{Availability of data and material}

The dataset supporting the conclusions of this article is included within the article (and its additional file.

\section{Competing interests}

The authors declare that they have no competing interest.

\section{Funding}

This study was not funded.

\section{Authors' contribution}

GM did conceptualization, analyzing the data, methodology designing, investigation during the laboratory work, writing original draft and review the final manuscript.

GF did conceptualization, analyzing the data, methodology designing, writing original draft and review the final manuscript.

TB did conceptualization, methodology designing, writing original draft and review the final manuscript. All authors have read and approved the manuscript.

\section{Acknowledgments}

The authors would like to thank all the study participants for their good collaboration. The authors would also like to thank the department of Medical Microbiology, School of Biomedical and Laboratory Science, College of Medicine and Health Sciences, University of Gondar for logistic and material supports. Finally, the authors would like to thank Amhara Public Health Institute for reagent support.

\section{References}

1. Lee AS, de Lencastre H, Garau J, Kluytmans J, Malhotra-Kumar S, Peschel A, et al. Methicillin-resistant Staphylococcus aureus. Nature reviews Disease primers. 2018;4:18033.

2. Sabbagh P, Riahi SM, Gamble HR, Rostami A. The global and regional prevalence, burden, and risk factors for methicillin-resistant Staphylococcus aureus colonization in HIV-infected people: A systematic review and meta-analysis. American journal of infection control. 2019;47(3):323-33.

3. Gupta S, Mishra B, Thakur A, Dogra V, Loomba PS, Jain M, et al. Risk factors associated with MRSA. Southern African Journal of Infectious Diseases. 2018;33(3):76-9. 
4. David MZ, Mennella C, Mansour M, Boyle-Vavra S, Daum RS. Predominance of methicillin-resistant Staphylococcus aureus among pathogens causing skin and soft tissue infections in a large urban jail: risk factors and recurrence rates. Journal of clinical microbiology. 2008;46(10):3222-7.

5. Haysom L, Cross M, Anastasas R, Moore E, Hampton S. Prevalence and risk factors for methicillinresistant Staphylococcus aureus (MRSA) infections in custodial populations: A systematic review. Journal of Correctional Health Care. 2018;24(2):197-213.

6. LDH. Methicillin Resistant Staphylococcus aureus (MRSA) in Prisons Louisiana Louisiana Department of Health [cited 2019 NOV. 23]. Avaliable from http://www.infectiousdisease.dhh.louisiana.gov/].

7. Köck R, Becker K, Cookson B, van Gemert-Pijnen J, Harbarth S, Kluytmans J, et al. Methicillin-resistant Staphylococcus aureus (MRSA): burden of disease and control challenges in Europe. 2010.

8. Chen C-J, Huang Y-C. New epidemiology of Staphylococcus aureus infection in Asia. Clin Microbiol Infect. 2014;20(7):605-23.

9. Zigmond J, Pecan L, Hájek P, Raghubir N, Omrani A. MRSA infection and colonization rates in Africa and Middle East: a systematic review \& meta-analysis. International Journal of Infectious Diseases. 2014;21:391.

10. Kejela T. The Spread of MRSA in Ethiopia. SCIOL Biomed. 2018;2:121-7.

11. Kejela T, Bacha K. Prevalence and antibiotic susceptibility pattern of methicillin-resistant Staphylococcus aureus (MRSA) among primary school children and prisoners in Jimma Town, Southwest Ethiopia. Ann Clin Microbiol Antimicrob. 2013;12(1):11.

12. Shibabaw A, Abebe T, Mihret A. Antimicrobial susceptibility pattern of nasal Staphylococcus aureus among Dessie Referral Hospital health care workers, Dessie, Northeast Ethiopia. International Journal of Infectious Diseases. 2014;25:22-5.

13. Tigabu A, Tiruneh M, Mekonnen F. Nasal carriage rate, antimicrobial susceptibility pattern, and associated factors of Staphylococcus aureus with special emphasis on MRSA among urban and rural elementary school children in Gondar, Northwest Ethiopia: A comparative cross-sectional study. Advances in preventive medicine.2018.

14. CSA E. Population projection of Ethiopia for all regions at wereda level from 2014-2017. Central Statistical Agency of Ethiopia. 2013.

15. The Federal Democratic Republic of Ethiopia CSA. National labour force survey [Internet]. Ethiopia: Central statistical agency of Ethiopia 2013 [cited 2019 Nov. 26]. Avaliable from .

16. Yamakhakha OK. Carriage of Staphylococcus aureus and methicillin-resistant Staphylococcus aureus (MRSA) among students of Jomo Kenyatta University of Agriculture and Technology, Kenya: JKUATCOHES. 2019.

17. Kubi A. Prevalence and antibiotic susceptibility pattern of methicillin resistant Staphylococcus aureus colonization among clinically tuberculosis suspected clients at St. Peter specialized hospital Addis Ababa Ethiopia: Addis Ababa Universty. 2018.

18. Cheesbrough M. District laboratory practice in tropical countries: Cambridge university press; 2006. 
19. CLSI. Performance standards for antimicrobial susceptibility testing: P. A. Wayne, Ed., Suppl. M100, Clinical and Laboratory Standards Institute, 28th edition; 2018.

20. Magiorakos AP, Srinivasan A, Carey RB, Carmeli Y, Falagas ME, Giske CG, et al. Multidrug resistant, extensively drug-resistant and pan drug-resistant bacteria: an international expert proposal for interim standard definitions for acquired resistance. Clinical Microbiology Infect. 2012;18(3):268-81.

21. Lowy FD. Staphylococcus aureus infections. New England journal of medicine. 1998;339(8):520-32.

22. DeLeo FR, Otto M, Kreiswirth BN, Chambers HF. Community-associated meticillin-resistant Staphylococcus aureus. The Lancet. 2010;375(9725):1557-68.

23. Sakr A, Brégeon F, Mège J-L, Rolain J-M, Blin O. Staphylococcus aureus nasal colonization: an update on mechanisms, epidemiology, risk factors, and subsequent infections. Frontiers in microbiology. 2018;9:2419.

24. Mukherjee D, Herzig C, Jeon C, Lee C, Apa Z, Genovese M, et al. Prevalence and risk factors for Staphylococcus aureus colonization in individuals entering maximum-security prisons. Epidemiology Infection. 2014;142(3):484-93.

25. Shuping LL, Kuonza L, Musekiwa A, lyaloo S, Perovic O. Hospital-associated methicillin-resistant Staphylococcus aureus: A cross-sectional analysis of risk factors in South African tertiary public hospitals. PloS one. 2017; 12(11).

26. Adam KM, Abomughaid MM. Prevalence of Methicillin-resistant Staphylococcus Aureus in Saudi Arabia Revisited: A Meta-analysis. The Open Public Health Journal. 2018; 11(1).

27. Cabrera EC, Ramirez-Argamosa DT, Rodriguez RD. Prevalence of community-acquired methicillinresistant Staphylococcus aureus from inmates of the Manila City Jail, characterization for SCCmec type and occurrence of Panton-Valentine leukocidin gene. Philipp Sci Letters. 2010;3(1):4-12.

28. Pan ES, Diep BA, Carleton HA, Charlebois ED, Sensabaugh GF, Haller BL, et al. Increasing prevalence of methicillin-resistant Staphylococcus aureus infection in California jails. Clin Infect Dis. 2003;37(10):1384-8.

29. Maree CL, Eells SJ, Tan J, Bancroft EA, Malek M, Harawa NT, et al. Risk factors for infection and colonization with community-associated methicillin-resistant Staphylococcus aureus in the Los Angeles County jail: a case-control study. Clin Infect Dis. 2010;51(11):1248-57.

30. Soltani B, Ardakani AT, Moravveji A, Erami M, Rezaei MH, Moniri R, et al. Risk factors for methicillin resistant Staphylococcus aureus nasal colonization of healthy children. Jundishapur journal of microbiology. 2014; 7(9).

31. Lekkerkerk W, Haenen A, van der Sande M, Leenstra T, de Greeff S, Timen A, et al. Newly identified risk factors for MRSA carriage in The Netherlands. PloS one. 2017; 12(11).

32. Reta A, Gedefaw L, Sewunet T, Beyene G. Nasal carriage, risk factors and antimicrobial susceptibility pattern of methicillin resistant Staphylococcus aureus among school children in Ethiopia. Journal of Medical Microbiology Diagnosis. 2015;4(1):1.

33. Kaleem F, Usman J, Hassan A, Omair M, Khalid A, Uddin R. Sensitivity pattern of methicillin resistant Staphylococcus aureus isolated from patients admitted in a tertiary care hospital of Pakistan. Iranian 
journal of microbiology. 2010;2(3):143.

34. Prabhu K, Chinniah TR, Ahmad RP, Bakar AA, Safar J. Inducible clindamycin resistance among Staphylococcus aureus isolates from skin and soft tissue infections: a study from Brunei Darussalam. Brunei international medical journal. 2016;11(5):235-40. 